\title{
Adaptação do Questionário de Tarefas Domésticas e de Cuidado de Irmãos
}

\author{
Adaptation of the Household Responsibilities \\ and Sibling Care Questionnaire
}

\author{
Letícia Lovato DELLAZZANA-ZANON ${ }^{1}$ \\ Cristian ZANON ${ }^{2}$
}

Lia Beatriz de Lucca FREITAS

\begin{abstract}
Resumo
O objetivo deste estudo foi adaptar o Household Responsibilities Questionnaire para avaliação de cuidado de irmãos e realização de tarefas domésticas por adolescentes brasileiros. Para isso, realizou-se a tradução e a adaptação dos itens para a população-alvo, seguindo as diretrizes de adaptação de testes da International Test Commission. Responderam à versão final do questionário 113 participantes com média de idade de 14,7 anos $(\mathrm{DP}=0,8)$, sendo $61,9 \%$ do sexo feminino. O conjunto de itens foi submetido a uma análise de eixos principais que revelou a solução bifatorial como mais apropriada. Os dois fatores, referentes a cuidado de irmãos e tarefas domésticas, apresentaram coeficientes alfa adequados de 0,75 e de 0,69 respectivamente. Esses resultados sugerem evidências de validade de construto para o questionário, assim como de fidedignidade. Assim, o questionário permite acessar esses fenômenos sistematicamente, o que pode ser fundamental para o avanço do conhecimento e de estimativas realizadas em nível nacional.
\end{abstract}

Palavras-chave: Gestão doméstica; Relações entre irmãos; Teste.

\begin{abstract}
The aim of this study was to adapt the Household Responsibilities Questionnaire for the assessment of sibling care and household responsibilities among Brazilian adolescents. To do this, we carried out the translation and adaptation of items for the target population following the test-adaptation guidelines of the International Test Commission. Participants were 113 adolescents (61.9\% female) with a mean age of 14.7 years (SD $=0.8)$ who answered the final version of the questionnaire. The set of items underwent principal axes analysis which revealed the two-factor solution as the most appropriate. The two factors, related to sibling care and household responsibilities, presented adequate alpha coefficients of 0.75 and 0.69 respectively. These results show evidence of construct validity for the questionnaire as well as reliability. Thus, this questionnaire enables systematic access to these phenomena, which can be critical to the advancement of knowledge and production of estimates at the national level.
\end{abstract}

Keywords: Household managements; Sibling relations; Testing.

$\operatorname{rrv}$

${ }^{1}$ Universidade Federal do Rio Grande do Sul, Instituto de Psicologia, Laboratório de Psicologia e Epistemologia Genética. R. Ramiro Barcelos, 2600, Sala 118, 90035-003, Porto Alegre, RS, Brasil. Correspondência para/Correspondence to: L.L. Dellazzana-Zanon. E-mail: <leticiadellazzana@gmail.com>.

2 Universidade São Francisco, Departamento de Psicologia, Programa de Pós-Graduação em Psicologia. Itatiba, SP, Brasil.

Artigo elaborado a partir da tese de L.L. Dellazzana-Zanon, intitulada "Projetos de vida na adolescência: comparação entre adolescentes que cuidam e que não cuidam de seus irmãos menores". Universidade Federal de Rio Grande do Sul, 2014.

Apoio: Coordenação de Aperfeiçoamento de Pessoal de Nível Superior. 
O trabalho realizado por jovens tem sido amplamente discutido pelas políticas públicas no Brasil e no mundo. Entretanto, existe um tipo de trabalho realizado por adolescentes que permanece praticamente invisível à sociedade: o cuidado exercido por irmãos mais velhos em relação aos mais novos. Embora exista um número relativamente pequeno de estudos sobre essa temática, o cuidado entre irmãos é descrito, em diferentes culturas, desde a década de 1970 (Carreño \& Avila, 2002; Dahlblom, Herrara, Pena, \& Dahlgren, 2009; East, Weisner, \& Slonim, 2009; Ferreira, 1991; Hafford, 2010; Weisner \& Gallimore, 1977).

É mais provável que o cuidado entre irmãos ocorra quando: (a) há muitos filhos na família, (b) o filho mais velho é do sexo feminino e (c) o filho mais novo já pode se locomover sozinho (Dunn, 1983). Além disso, em algumas culturas, o cuidado de crianças pequenas não é considerado uma tarefa dos pais, mas sim dos irmãos mais velhos (Weisner \& Gallimore, 1977). Resultados de pesquisas indicam que, em famílias de nível socioeconômico baixo - principalmente as monoparentais -, a ausência de um dos progenitores pode exigir que os irmãos mais velhos cuidem dos mais novos (Dahlblom et al., 2009; East et al., 2009; Hafford, 2010). Sabe-se que o cuidado entre irmãos é uma forma encontrada pelas famílias de nível socioeconômico baixo para dar conta do cuidado (Burton, 2007; Fulighi, Hughes, \& Way, 2009; Poletto \& Koller, 2008) e da sobrevivência de seus membros mais novos (Amazonas, Damasceno, Terto, \& Silva, 2003). Com o intuito de avaliar apropriadamente o quanto adolescentes cuidam de seus irmãos menores e realizam tarefas domésticas, este estudo tem por finalidade adaptar uma escala que acesse esses fenômenos de forma rápida e válida.

No que se refere às estatísticas nacionais oficiais, pode-se encontrar referência ao cuidado de crianças na categoria afazeres domésticos (Instituto Brasileiro de Geografia e Estatística [IBGE], 2010). Tal categoria está relacionada às tarefas e ao trabalho realizado no próprio domicílio de residência. De acordo com a Pesquisa Nacional por Amostragem de Domicílios (PNAD) (IBGE, 2010), inclui478 -se nessa categoria o cumprimento das seguintes tarefas realizadas no domicílio: (a) arrumar ou limpar toda ou parte da moradia; (b) cozinhar ou preparar alimentos, passar roupa, lavar roupa ou louça, utilizando ou não aparelhos eletrodomésticos para executar estas tarefas para si ou para outro(s) morador(es); (c) orientar ou dirigir trabalhadores na execução das tarefas domésticas; (d) cuidar de filhos ou menores de idade; e (e) limpar o quintal ou terreno que circunda a residência.

Soares e Sabóia (2007) chamam atenção para o fato de que o conceito utilizado pela PNAD é muito amplo e não permite identificar quais tarefas são efetivamente realizadas, uma vez que, ao citar apenas uma delas, considera-se que a pessoa realize afazeres domésticos. Mesmo com essa limitação, dados de pesquisas do IBGE (2008) indicam que houve um aumento na proporção de crianças que realizavam trabalho doméstico entre 1997 $(5,4 \%)$ e 2007 (8,0\%).

Assim como ocorre com a definição de afazeres domésticos do IBGE, a maior parte dos estudos nacionais que mencionam o trabalho realizado em casa indica que as atividades de trabalho referem-se à realização de tarefas domésticas, entre as quais se incluem tarefas de cuidado dos irmãos mais novos (Bem \& Wagner, 2006; Bezerra, 2006; Kosminsky \& Santana, 2006). Ainda em relação a pesquisas realizadas no Brasil, poucos são os estudos que enfocaram especificamente o cuidado entre irmãos (Dellazzana, 2008; Dellazzana \& Freitas, 2010; Ferreira, 1991; Ferreira \& Mettel, 1999; Poletto, Wagner, \& Koller, 2004), sendo que todos eles são qualitativos.

Os trabalhos de Ferreira (1991) e Ferreira e Mettel (1999) apresentam resultados de uma pesquisa cujo objetivo foi estudar a interação entre irmãos e o desempenho nas tarefas domésticas executadas por irmãos mais velhos responsáveis pelos cuidados dos mais novos durante a ausência dos pais. Participaram 20 famílias de nível socioeconômico baixo, com filhos entre um e 16 anos de idade. A coleta de dados foi realizada por meio de entrevistas e observação direta do comportamento das famílias.

Os resultados mostraram que: (a) o cuidado dos irmãos menores pelos irmãos mais velhos foi 
favorecida pelo grande número filhos, (b) há correspondência entre comportamentos de cuidado entre irmãos e intervalo amplo de idades entre eles; (c) o principal motivo para os filhos assumirem a responsabilidade pelas tarefas domésticas foi a saída da mãe para o mercado de trabalho, e (d) a maior parte dos participantes começou a realizar tarefas domésticas e de cuidados dos irmãos menores auxiliando suas mães desde muito cedo, ao redor de cinco anos de idade (Ferreira, 1991; Ferreira \& Mettel, 1999).

Observou-se, além disso, que a funcionalidade das famílias nas quais um irmão mais velho realiza tarefas domésticas está relacionada com dados sociodemográficos, organização familiar e rede social de apoio, uma vez que tais aspectos interferem nas práticas educativas das crianças. Ferreira (1991) assinala que, embora se saiba que atividades de cuidado entre irmãos em situações de brincadeira e de auxílio às mães sejam consideradas vantajosas para os irmãos, os efeitos do cuidado sistemático ainda precisam ser investigados.

Em outro estudo, Poletto et al. (2004) investigaram, à luz do construto de resiliência, como meninas de nível socioeconômico baixo lidam com a responsabilidade pelo cuidado de irmãos menores como parte dos cuidados da casa, o que foi considerado pelas autoras como uma situação de risco. Participaram dessa investigação quatro meninas com idade entre 8 e 12 anos, as quais possuíam entre um e 3 irmãos mais novos. Todas as meninas permaneciam em casa no período extraescolar, no qual realizavam atividades domésticas e tinham a responsabilidade de cuidar de irmãos menores. Os dados foram coletados individualmente, mediante uma entrevista semiestruturada.

Os principais resultados mostraram que as meninas realizam tarefas domésticas, que incluem limpar e arrumar a casa, lavar a roupa, preparar as refeições da família, além de responsabilizar-se pelos cuidados dos irmãos mais novos. Assim, evidenciou-se que as participantes: (a) dedicam grande parte do seu tempo às atividades da casa e ao cuidado dos irmãos menores, (b) não frequentam mais o centro de atendimento à criança do bairro em razão da falta de tempo, e (c) têm pouco espaço para brincadeiras em sua rotina. Por outro lado, observou-se que a relação com os irmãos parece ser saudável, uma vez que a maioria das participantes relatou se sentir bem na companhia deles e compartilhar momentos de brincadeiras (Poletto et al., 2004).

Poletto et al. (2004) observaram que, em razão das atividades que precisam realizar quanto ao cuidado dos irmãos e da casa, as participantes do estudo são forçadas a amadurecer mais cedo, quando comparadas a outras crianças da mesma idade que não vivenciam essas situações. Para dar conta da sobrecarga e das adversidades de sua rotina, as meninas são obrigadas a buscar recursos, fazendo uso da resiliência, o que contribui no enfrentamento e na superação de problemas. Todavia, nem sempre as meninas possuem recursos suficientes e apoio para dar conta de todas as demandas do meio, o que pode causar dano psicológico.

Outro estudo realizado recentemente (Dellazzana, 2008; Dellazzana \& Freitas, 2010) teve como objetivo descrever a rotina de adolescentes de baixa renda em situação de vulnerabilidade social. Os participantes dessa pesquisa foram 20 adolescentes com idade entre 12 e 16 anos, sendo 16 do sexo feminino e 4 do masculino. Os participantes tinham entre dois e oito irmãos. Os dados foram coletados individualmente mediante ficha de dados sociodemográficos, genograma familiar e entrevista semiestruturada sobre um dia de sua vida. Dentre os principais resultados do estudo, dois aspectos merecem ser destacados: (a) o fato de que o cuidado entre irmãos pode ser formal ou informal, e (b) a ideia de que, embora possam estar associadas, atividades domésticas e atividades de cuidado constituem grupos distintos de tarefas, uma vez que podem ou não coexistir. Em outras palavras, alguns adolescentes realizam tarefas domésticas mas não cuidam de irmãos, enquanto outros cuidam dos irmãos mas não realizam tarefas domésticas. Assim, acredita-se que tarefas domésticas e cuidados entre irmãos podem representar dimensões com algum grau de independência, o que será avaliado empiricamente na análise da estrutura do questionário.

Observaram-se dois tipos de cuidado entre irmãos. O cuidado informal acontece quando o 
adolescente auxilia a mãe a cuidar das crianças mais novas, por exemplo, enquanto ela está fazendo o almoço ou arrumando a casa. Deve-se ressaltar que, no caso das famílias nucleares e reconstituídas, nenhum participante mencionou ajudar o pai, ao se referir ao cuidado dos irmãos e às atividades domésticas. A figura materna está sempre presente e consegue assumir a tarefa de cuidar de todos os filhos. Nesse caso, o filho mais velho parece não sofrer prejuízos em seu desenvolvimento, em função do cuidado dos irmãos menores. Já o cuidado formal ocorre quando o adolescente é o responsável por cuidar dos irmãos mais novos. Embora exista a figura materna, não é ela quem assume a tarefa de cuidar dos filhos mais novos. As tarefas de cuidado dos irmãos mais novos são a principal atividade que o filho mais velho desempenha ao longo do dia (Dellazzana, 2008; Dellazzana \& Freitas, 2010).

Quanto às atividades que os adolescentes cuidadores executam ao longo do dia, podem-se citar: (a) servir o café da manhã, o almoço, o café da tarde, o jantar, e dar mamadeira, (b) dar banho, vestir e pentear, (c) levar e buscar na escola e nas atividades extraclasse, (d) colocar os irmãos para assistir televisão, fazer dormir, trocar as fraldas, e (e) cuidar dos irmãos enquanto eles estão brincando. Entretanto, não é apenas com o cuidado dos irmãos menores que o adolescente cuidador se envolve. Essa mesma pesquisa evidenciou que, além das atividades de cuidado dos irmãos mais novos, esses adolescentes também realizam tarefas domésticas, que incluem: (a) fazer o café da manhã, lavar a louça do café da manhã, fazer o almoço, lavar a louça do almoço, fazer o café da tarde, limpar o fogão e a cozinha, (b) varrer a sala, varrer o chão da casa e o do pátio, (c) arrumar as camas, as cobertas, limpar os quartos, e (d) lavar e estender as roupas.

Quando se leva em conta os adolescentes caracterizados como cuidadores formais, a realização de atividades de cuidado dos irmãos menores e atividades domésticas acarreta uma série de prejuízos para eles. Eles sofrem um prejuízo importante em relação à escola, uma vez que são infrequentes e não têm tempo para realizar temas de casa (em Porto Alegre, a expressão "tema de casa" refere- em casa). Observou-se que eles apresentam uma defasagem escolar significativa, pois a maior parte dos participantes desse estudo já sofreu até três reprovações. Além disso, os adolescentes cuidadores também têm pouco tempo para o lazer (Dellazzana, 2008; Dellazzana \& Freitas, 2010).

Na realidade brasileira, a falta de vagas nas escolas públicas de educação infantil deve ser também considerada para se compreender por que adolescentes assumem papel de cuidadores de seus irmãos (Dellazzana \& Freitas, 2010). Pesquisas recentes revelam que há uma discrepância entre o número de crianças com idade para frequentar creches e o número real de vagas disponíveis, principalmente em relação às crianças de zero a 3 anos de idade (Freitas, Shelton, \& Sperb, 2009; Freitas, Shelton, \& Tudge, 2008). Soma-se a isso o fato de que muitas famílias em situação de vulnerabilidade social, que sobrevivem apenas com os benefícios de programas sociais, são excluídas do sistema de educação infantil, visto que muitas creches da rede pública priorizam crianças cujas mães trabalham fora de casa (Dellazzana \& Freitas, 2010).

Como o cuidado entre irmãos é um fenômeno praticamente invisível e, na maioria das vezes, considerado um tipo de trabalho doméstico, a existência de um instrumento padronizado poderia auxiliar pesquisadores a acessar tanto atividades de cuidado entre irmãos quanto de tarefas domésticas de forma rápida e objetiva. Além disso, o uso de um questionário válido e fidedigno possibilitaria a comparação de médias entre diferentes grupos e poderia ajudar a mapear áreas com alta concentração dessas tarefas entre os adolescentes. Uma vez que tais práticas podem estar associadas à evasão e ao baixo desempenho escolar, a avaliação adequada do fenômeno pode ser o primeiro passo para o desenvolvimento de intervenções que visem à melhoria das condições de vida desses jovens.

Considerando-se que a existência de um instrumento que permita estudar o fenômeno sistematicamente pode ser fundamental para o avanço do conhecimento sobre o tema, bem como para ajudar a melhorar estimativas realizadas em nível nacional sobre afazeres domésticos, viu-se no instrumento original - Household Responsibilities Questionnaire 
(Riggio, Valenzuela, \& Weiser, 2010) -, uma possibilidade de acessar adolescentes que cuidam dos irmãos menores, mediante algumas adaptações. Dessa forma, este artigo tem como objetivos: (a) realizar a adaptação do Household Responsibilities Questionnaire para a avaliação do cuidado de irmãos menores e de tarefas domésticas e (b) avaliar evidências de validade de construto e fidedignidade dos escores da escala adaptada. Entende-se por adaptação de instrumento todas as atividades - incluindo a tradução -, usadas para decidir se um instrumento é ou não apropriado para avaliar o mesmo construto em uma língua ou cultura diferente (Hambleton, 2005; International Test Commission [ITC], 2010). O processo de adaptação de instrumento inclui: (a) a seleção de tradutores capazes, (b) as decisões sobre quais adequações são necessárias para o uso do instrumento em uma segunda língua, e (c) a avaliação da versão adaptada em face do original (Hambleton, 2005).

\section{Método}

\section{Participantes}

Participaram deste estudo 114 adolescentes de sete escolas municipais, contemplando as quatro regiões da rede municipal de ensino de Porto Alegre. Os critérios de inclusão dos participantes da amostra foram: (a) ter entre 14 e 16 anos, (b) ser aluno da rede municipal de ensino, e (c) ter no mínimo um irmão mais novo coabitando. Optou-se por escolas municipais, uma vez que são elas que atendem a maior número de jovens oriundos de famílias de nível socioeconômico baixo, nas quais o cuidado entre irmãos é mais comum. A amostra foi de conveniência, e a participação dos adolescentes foi voluntária.

Uma participante foi excluída da amostra, pois sua irmã mais velha já havia participado da pesquisa. A média de idade dos 113 participantes foi de 14,7 (Desvio-Padrão-DP $=0,8$ ), sendo que $38,9 \%$ eram do sexo masculino e $61,9 \%$ do feminino. Constatou-se $4,0 \%$ de missing data, que foram substituídos usando o método de multiple regression imputation (Byrne, 2006). Os itens referentes a cuidados com irmão foram usados como preditores para os missing data verificados nesse conjunto de itens, e itens referentes a cuidados domésticos foram usados como preditores para os missing data desse conjunto de itens.

\section{Instrumentos}

Utilizaram-se dois instrumentos: (a) um questionário com questões sociodemográficas elaborado a fim de caracterizar a amostra apropriadamente, e (b) o Questionário de Tarefas Domésticas e de Cuidado de Irmãos (QTDCI), que está sendo adaptado neste trabalho.

\section{Procedimentos}

O Questionário de Tarefas Domésticas e de Cuidado de Irmãos (QTDCI) foi desenvolvido a partir da adaptação do Household Responsibilities Questionnaire (Riggio et al., 2010). O questionário original, desenvolvido para estudantes universitários, é composto por 14 itens que investigam a realização de responsabilidades domésticas, sendo integrado por: (a) 8 itens que acessam a frequência com a qual os participantes se envolveram em diferentes tarefas domésticas ao longo do seu crescimento (lavar louça, lavar roupa, lavar, varrer/aspirar o piso, limpar as janelas, tirar pó dos móveis, limpar banheiro, levar o lixo para a rua), (b) 2 itens sobre a frequência da preparação de refeições para eles mesmos ou para outros membros da família, (c) 1 item sobre a frequência com a qual os participantes realizam atividades de rua para os pais (como pagar uma conta), (d) 1 item sobre a frequência com a qual cuidam de si mesmos, e (e) 2 itens sobre cuidado de irmãos menores. Cada item foi respondido em uma escala de tipo Likert de frequência de cinco pontos, na qual os extremos são "nunca" (1) e "quase todos os dias" (5).

Além desses 14 itens, outros três solicitavam ao participante que indicasse: (a) se ele realizava tarefas domésticas diárias ao longo do seu crescimento/desenvolvimento, assinalando sim ou não, 
(b) a idade que tinha quando começou a realizar tarefas domésticas diárias ou semanais, e (c) a idade que tinha quando começou a tomar conta de si mesmo sozinho. Apesar de bastante informativos, esses itens foram traduzidos, mas não incluídos nas análises de agrupamento de dados, uma vez que eles remetem a questões do passado do sujeito, não necessariamente refletindo a atual situação de trabalho e cuidado. Ressalta-se, entretanto, que eles foram mantidos por contribuírem com detalhes importantes sobre o respondente. A seguir, são descritos os procedimentos de tradução e adaptação do instrumento.

Inicialmente, o questionário foi traduzido para o português brasileiro por uma pessoa bilíngue. Um falante nativo da língua inglesa foi consultado para auxiliar na compreensão da expressão "run errands", sem correspondente em português, a qual foi adaptada para "fazer tarefas de rua para os seus pais". Um exemplo (fazer compras) foi acrescentado para facilitar a compreensão do item pelos participantes.

Na versão original do questionário, o foco de interesse dos pesquisadores era o período em que o participante estava se desenvolvendo - a infância e a adolescência -, portanto, a referência para os participantes era "while you were growing up" e os itens estavam no tempo verbal passado. Com o intuito de investigar as experiências recentes em relação ao cuidado dos irmãos, optou-se por usar o tempo verbal presente, sem referência específica a algum período de tempo.

Outra alteração realizada diz respeito a dois itens do questionário original: um item que tratava da frequência com a qual o participante lavava o chão e outro sobre a frequência com a qual ele aspirava o piso foram condensados em um único indicador que avalia a frequência com a qual o participante limpa o piso/chão da casa. Esses ajustes estão de acordo com a recomendação D. 2 das diretrizes de adaptação de testes da International Test Commission (ITC, 2010), a qual prevê alterações no conteúdo dos itens com a finalidade de se adequar à população-alvo.

A chave de respostas da escala do questioná482 rio também foi alterada, visto que se considerou que a utilização de apenas valores extremos como âncoras poderia ser inapropriada para esse grupo, aumentando a imprecisão atribuída aos valores intermediários assinalados pelos participantes. Para reduzir esse viés, todos os pontos da chave de respostas apresentaram uma frequência associada. Por exemplo, "2" significa um ou dois dias na semana, "3" significa três ou quatro dias na semana. Os demais pontos são apresentados na escala (Anexo). Essa alteração está de acordo com a recomendação D.3 das diretrizes da ITC (2010), que sugere adaptação do sistema de respostas dos itens quando ele for inadequado ou puder ser fonte de viés para a população-alvo. Apesar de o sistema de respostas original não ser totalmente inadequado, ele é pouco preciso para a amostra de adolescentes em questão, já que foi elaborado para uma amostra de universitários americanos. Uma vez que a amostra deste estudo é composta por um grupo com baixa escolaridade, optou-se por definir todos os pontos da escala de respostas, a fim de facilitar a compreensão do significado dos valores associados.

A seguir, realizou-se uma tradução reversa do questionário por outra pessoa bilíngue. A versão original e a tradução reversa foram comparadas por uma pessoa bilíngue independente, expert no tema de cuidado de irmãos e tarefas domésticas. Apesar de os tradutores bilíngues não apresentarem o mesmo nível de fluência em ambas as línguas, eles estavam familiarizados com o tema e atendiam ao critério de bilíngues funcionais. Não foram verificadas incompatibilidades significativas entre as versões para além das alterações descritas, o que indica que a tradução foi adequada.

Uma análise do conteúdo dos itens sugeriu que aquele referente a "cozinhar para si mesmo" fosse excluído do questionário, pois se considerou que, em famílias com muitos membros (muitos filhos/crianças), essa pergunta não faria sentido. Ao contrário do que ocorre nas famílias de classe média e alta, elas são regidas pela lógica da solidariedade, pela qual o importante é a manutenção do grupo familiar como um todo (Amazonas et al., 2003). Deve-se levar em conta, também, o fato de que o instrumento original foi desenvolvido para universitários americanos, os quais, em geral, moram so- 
zinhos ou com colegas - e não com suas famílias de origem, como é o caso dos adolescentes participantes deste estudo. Nesse sentido, considerou-se "cozinhar para si mesmo" um comportamento que raramente seria observado.

Uma vez que um dos objetivos principais deste questionário foi avaliar a frequência com que irmãos mais velhos cuidam dos menores, foram adicionados os seguintes itens: (a) frequência com a qual dá banho nos irmãos menores, (b) frequência com a qual leva ou busca os irmãos menores na escola ou em outra atividade extraclasse, e (c) frequência com a qual serve comida aos irmãos menores. Além disso, foi incluído um item sobre a idade que o participante tinha quando começou a cuidar dos irmãos menores. Todos esses itens basearam-se nos resultados de uma pesquisa que investigou o cotidiano de adolescentes que cuidam dos irmãos menores (Dellazzana, 2008; Dellazzana \& Freitas, 2010) e que se mostraram indicativos importantes sobre a ocorrência de cuidado entre irmãos. Devido a todas as alterações já descritas, esta adaptação não caracteriza um equivalente do instrumento original e, portanto, comparações diretas por meio de testes estatísticos não são recomendadas.

Os procedimentos para a coleta de dados foram os seguintes: (a) apresentação do projeto de pesquisa e solicitação de autorização para a participação das escolas municipais à coordenadora do Ensino Médio da Secretaria Municipal de Educação de Porto Alegre, (b) indicação de escolas nas quais haveria maior probabilidade de encontrar o perfil de adolescentes almejado, (c) contato com cada uma das escolas indicadas a fim de agendar uma reunião para apresentação do estudo e pedido de autorização, (d) entrega, assinatura e devolução do Termo de Consentimento Livre e Esclarecido (TCLE) dos alunos que preenchiam os critérios de inclusão, bem como seus pais ou responsáveis legais e (e) agendamento da coleta de dados.

Os Termo de Consentimento Livre e Esclarecido foram entregues aos alunos, solicitando-lhes que os pais ou responsáveis os assinassem e devolvessem na escola. Os diretores e funcionários das escolas nas quais a coleta foi realizada referiram que seria muito difícil que os termos retornassem assinados, pois há uma questão cultural nas famílias da comunidade de não dar retorno por escrito às solicitações da escola, de uma maneira geral. De fato, isso foi confirmado, uma vez que somente $25 \%$ deles retornaram. Optou-se, então, por outra estratégia: entregar os TCLE primeiro aos progenitores e depois aos alunos. Como a coleta de dados foi realizada em um período próximo às entregas de avalições dos alunos (às quais os pais ou responsáveis precisam estar presentes), a pesquisadora decidiu comparecer às escolas nesse dia e abordá-los diretamente. Nesse momento, explicava-se a pesquisa e solicitava-se a autorização e a assinatura do TCLE. Essa maneira de obter a assinatura do TCLE mostrou-se muito mais eficaz, pois, além de conversar diretamente com os responsáveis para explicar a pesquisa, era possível responder a suas dúvidas. Caso eles autorizassem a participação do filho, este também podia assinar o TCLE.

Priorizou-se a aplicação coletiva dos instrumentos, a qual foi realizada no mesmo turno em que os adolescentes estudavam. Em geral, a aplicação ocorria com um grupo pequeno de estudantes, aproximadamente quatro alunos. Entretanto, em duas ocasiões, a aplicação foi realizada com um grupo de 15 alunos. Duas participantes não sabiam ler. Nesses casos, leu-se o instrumento e marcaram-se as questões de acordo com aquilo que as participantes responderam.

O projeto de pesquisa foi aprovado pelo Comitê de Ética em Pesquisa do Instituto de Psicologia da Universidade Federal do Rio Grande do Sul (Protocolo $n^{\circ} 20849$ ).

\section{Resultados e Discussão}

Neste artigo, descrevem-se os procedimentos de adaptação do Household Responsibilities Questionnaire para adolescentes de famílias de baixa renda e apresentam-se evidências de validade de construto e fidedignidade dos escores do questionário adaptado. Durante a coleta de dados, alguns participantes relataram que suas casas não tinham vidros (alusão ao item do questionário que tratava da frequência com que eles limpavam os 
vidros das janelas), e que eles não costumavam fazer atividades fora de casa (como pagar contas) para seus pais. Devido a essas constatações, optou-se por não incluir tais itens na análise de dados.

Com intuito de estabelecer a melhor forma de pontuar o questionário, ou seja, avaliar como os itens se agrupam, o conjunto deles foi submetido a uma análise de eixos principais com rotação oblimin. Essa rotação foi escolhida porque se conjecturou que, apesar de trabalho doméstico e cuidado entre irmãos serem dimensões distintas, elas não são ortogonais.

Na primeira extração, verificaram-se os dois fatores esperados (cuidado entre irmãos e tarefas domésticas); contudo, o item referente a lavar louça apresentou carga fatorial de aproximadamente 0,30 em ambas as dimensões. Uma vez que ele obteve baixa comunalidade, ou seja, pouca relação com o conjunto total de itens, optou-se por eliminá-lo. A segunda extração também apresentou uma solução interpretável em que se verificou: índice Kaiser-Meyer-Olkin de 0,78 e teste de esfericidade de Bartlett significativo $(p<0,001)$. Esses resultados sugerem adequação dos dados para a análise. Nesta solução, apenas dois fatores apresentaram eigenvalue maior que 1 , o que sugere que o conjunto de itens se agrupa em dois fatores.

O primeiro fator, composto por cinco itens referentes a trabalho doméstico, apresentou um eingenvalue de 3,3, que explicou 37,0\% de variância. O segundo fator, composto por quatro itens referen-tes a cuidado com irmão, apresentou um eingenvalue de 1,3, que explicou $14,7 \%$ de variância. Todos os itens apresentaram carga fatorial superior a 0,37 no fator esperado e cargas abaixo de 0,23 no outro fator. a de dois fatores é coerente com as expectativas de que trabalho doméstico e cuidado entre irmãos são dimensões que se relacionam, embora sejam distintas. Essa constatação indica evidências de validade de construto para o questionário, uma vez que tal distinção já fora verificada em outro estudo (Dellazzana, 2008). A consistência interna do primeiro fator foi de 0,75 , enquanto a do segundo foi de 0,69, o que é aceitável para pesquisa.
Verificaram-se diferenças significativas nas médias de frequência com que se realizaram tarefas domésticas entre meninas $(M=13,4 ; D P=4,9)$ e meninos $(M=10,5 ; D P=4,2)$, $[t(100)=3,2$; $p<0,01 ; d=0,6]$. $O$ " $d$ " é uma estimativa de tamanho de efeito em termos de desvios-padrão. Neste caso, indica que a média de meninas está 0,6 desvios-padrão acima da média dos meninos (Cohen, 1998). Esses resultados corroboram dados de pesquisas (Bruschini, 2007; Lago, Souza, Kaszubowski, \& Soares, 2009; Soares \& Sabóia, 2007), bem como de estatísticas nacionais oficiais (IBGE, 2008), as quais referem que mulheres (independentemente da idade) continuam exercendo atividades domésticas com maior frequência do que homens. Segundo um estudo sobre tempo, trabalho e afazeres domésticos com base nos dados da PNAD de 2001 e 2005, "afazeres domésticos constituem um grupo de atividades predominantemente femininas" (Soares \& Sabóia, 2007, p.10).

Quanto ao cuidado dos irmãos menores, não foram verificadas diferenças entre os gêneros. Esse dado contraria resultados de outros estudos realizados sobre o tema, os quais referem que relações de cuidado entre irmãos são mais frequentes quando o cuidador é do sexo feminino (Dunn, 1983; Ferreira, 1991; Lordelo \& Carvalho, 1999).

A partir da análise fatorial, foi possível verificar que o QTDCI, de fato, avalia o que ele se propõe avaliar. A verificação de dois fatores como solução mais apropriada corrobora as expectativas do estudo e apresenta evidências de validade de construto para ambos os fatores do questionário. Uma vez que os dois fatores apresentaram coeficientes alfa adequados, pode-se dizer que o QTDCI avalia os dois construtos com precisão. Nesse sentido, uma contribuição importante deste estudo é distinguir dois aspectos - tarefas domésticas e cuidado entre irmãos -, que tanto a literatura quanto as estatísticas nacionais oficiais entendem como sendo parte do mesmo construto.

Uma vez que existem graus de intensidade em relação ao cuidado dos irmãos menores e que, quanto maior o tempo empregado no cuidado destes, mais prejuízos os adolescentes cuidadores podem sofrer, faz-se necessária uma escala capaz 
de medir o fenômeno. Quanto a sua importância em relação à pesquisa, o QTDCI poderá contribuir para o avanço do conhecimento sobre o tema, além de diferenciar cuidado entre irmãos de outras tarefas domésticas, ajudando, assim, a aprimorar a coleta de dados para as estimativas realizadas no país sobre afazeres domésticos.

Além disso, considerando-se que situações de cuidado dos irmãos menores são de pouca visibilidade, a utilização do QTDCI poderá auxiliar psicólogos e assistentes sociais que trabalham com famílias de baixa renda a identificarem a ocorrência do fenômeno. Assim, a situação de cuidado entre irmãos poderá ser reconhecida e divulgada, a fim de que intervenções particulares com cada família (quando for o caso) sejam realizadas, para: (a) pensar formas alternativas de encontrar cuidadores para os membros mais novos das famílias, e (b) diminuir as possíveis consequências negativas do fenômeno no desenvolvimento dos adolescentes.

Uma vez que os participantes são oriundos apenas da capital de um estado da região sul do país, esses resultados não devem ser generalizados para toda a população brasileira e precisam ser interpretados com cautela. Por isso, sugere-se que novos estudos sejam realizados com essa escala em outras partes do país, a fim de verificar a adequação (ou não) das propriedades psicométricas do questionário para outras regiões.

Deve-se ressaltar ainda que este artigo apresentou um procedimento de coleta de dados inovador para as situações nas quais pais ou responsáveis devem assinar o TCLE em pesquisas que ocorrem na escola. Abordar os pais no momento em que eles estão na escola para a entrega das avaliações dos filhos mostrou-se uma forma eficiente de conseguir autorização para participação na pesquisa. Tal procedimento poderá ser usado por outros pesquisadores para a obtenção de autorização para menores de idade. Por fim, apesar de a intenção deste artigo não ser indicar qual é a forma mais adequada de avaliar cuidado entre irmãos e realização de tarefas domésticas, o uso de um questionário breve, como o aqui apresentado, possibilita que o fenômeno seja avaliado em grandes grupos de forma válida e rápida.

\section{Referências}

Amazonas, M. C. L. A., Damasceno, P. R., Terto L. M. S., \& Silva, R. R. (2003). Arranjos familiares de crianças das camadas populares. Psicologia em Estudo, 8(Esp.), 11-20.

Bem, L. A., \& Wagner, A. (2006). Reflexões sobre a construção da parentalidade e o uso de estratégias educativas em famílias de baixo nível socioeconômico. Psicologia em Estudo, 11(1), 63-71.

Bezerra, M. E. G. (2006). O trabalho infantil afeta o desempenho escolar no Brasil? (Dissertação de mestrado não-publicada). Universidade de São Paulo, Piracicaba.

Bruschini, M. C. A. (2007). Trabalho e gênero no Brasil nos últimos dez anos. Cadernos de Pesquisa, 37(132), 537-572.

Burton, L. (2007). Childhood adultification in economically disadvantaged families: A conceptual model. Family Relations, 56(4), 329-345.

Byrne, B. M. (2006). Structural equation modeling with EQS: Basic concepts, applications, and programming. London: Routledge.

Carreño, C. M., \& Avila, S. C. (2002). El vínculo de apego entre hermanos: um estudio exploratório con ninõs colombianos de estrato bajo. Suma Psicologica, 9(1), 107-132.

Cohen, J. (1988). Statistical power analysis for the behavioral sciences ( $2^{\text {nd }}$ ed.). Hillsdale: Erlbaum.

Dahlblom, K., Herrara, A. R., Pena, R., \& Dahlgren, L. (2009). Home alone: Children as caretakers in León, Nicarágua. Children \& Society, 23(1), 43-56.

Dellazzana, L. L. (2008). Irmãos que cuidam de irmãos: concepções sobre justiça retributiva (Dissertação de mestrado não-publicada). Universidade Federal do Rio Grande do Sul, Porto Alegre.

Dellazzana, L. L., \& Freitas, L. B. L. (2010). Um dia na vida de irmãos que cuidam de irmãos. Psicologia: Teoria e Pesquisa, 26(4), 595-603.

Dunn, J. (1983). Sibling relationships in early childhood. Child Development, 54(4), 787-811.

East, P. L., Weisner, T. S., \& Slonim, A. (2009). Youths' caretaking of their adolescent sisters' children: Results from two longitudinal studies. Journal of Family Issues, 30(12), 1671-1697.

Ferreira, E. A. P. (1991). Irmãos que cuidam de irmãos na ausência dos pais: um estudo sobre desempenho em tarefas domésticas e interação ente irmãos (Dissertação de mestrado não-publicada). Universidade de Brasília.

Ferreira, E. A. P., \& Mettel, T. P. L. (1999). Interação entre irmãos em situação de cuidados formais. Psicologia: Reflexão e Crítica, 12(1), 133-146. 
Freitas, L. B. L., Shelton, T. L., \& Sperb, T. M. (2009). Conceptions of early childhood care and education in Brazil. In M. Fleer, M. Hedegaard, \& J. Tudge (Eds.), World Yearbook of Education 2009: Childhood studies and the impact of globalization: Policies and practices at global and local levels (pp.279-291). London: Routledge.

Freitas, L. B. L., Shelton, T. L., \& Tudge, J. R. H. (2008). Conceptions of US and Brazilian early childhood care and education: A historical and comparative analysis. International Journal of Behavioral Development, 32(2), 161-170.

Fulighi, A. J., Hughes, D. L., \& Way, N. (2009). Ethnicity and immigration. In R. M. Lerner \& L. Steinberg (Eds.), Handbook of adolescent psychology: Contextual influences on adolescent development (Vol. 3, pp.527569). Hoboken (NJ): Wiley. Retrieved January 15, 2012, from http://books.google.com/books

Hafford, C. (2010). Sibling caretaking in immigrant families: Understanding cultural practices to inform child welfare practice and evaluation. Evaluation and Program Planning, 33(3), 294-302.

Hambleton, R. K. (2005). Issues, designs, and technical guidelines for adapting tests in multiple languages. In R. K. Hambleton, P. Merenda, \& C. Spielberger (Eds.), Adapting educational and psychological tests for cross-cultural assessment (pp.3-38). Hillsdale (MI): Lawrence Erlbaum Associates.

Instituto Brasileiro de Geografia e Estatística. (2008). Síntese de indicadores sociais: uma análise das condições de vida da população brasileira. Rio de Janeiro: IBGE.

Instituto Brasileiro de Geografia e Estatística. (2010). Pesquisa nacional por amostra de domicílios: síntese de indicadores 2009. Rio de Janeiro: IBGE.

International Test Commission. (2010). International Test Commission Guidelines for translating and adapting tests. Liverpol: International Test Commission. Retrieved May 10, 2012, from http://www.intestcom.org

Kosminsky, E. V., \& Santana, J. N. (2006). Crianças e jovens e o trabalho doméstico: a construção social do feminino. Sociedade e Cultura, 9(2), 227-236.

Lago, M. C. S., Souza, C. D., Kaszubowski, E., \& Soares, M. S. (2009). Gênero, gerações e espaço doméstico: trabalho, casa e família. Paidéia, 19(44), 357-366.

Lordelo, E. R., \& Carvalho, A. M. A. (1999). Um estudo naturalístico do comportamento de cuidado entre crianças pré-escolares. Biotemas, 12(1), 7-30.

Poletto, M., \& Koller, S. H. (2008). Contextos ecológicos: promotores de resiliência, fatores de risco e de proteção. Estudos de Psicologia (Campinas), 25(3), 405-416.

Poletto, M., Wagner, T. M. C., \& Koller, S. H. (2004). Resiliência e desenvolvimento infantil de crianças que cuidam de crianças: uma visão em perspectiva. Psicologia: Teoria e Pesquisa, 20(3), 241-250.

Riggio, H. R., Valenzuela, A, M., \& Weiser, D. A. (2010). Household responsibilities in the family of origin: Relations with self-efficacy in young adulthood. Personality and Individual Differences, 48(5), 568-573.

Soares, C., \& Sabóia, A. L. (2007). Tempo, trabalho e afazeres domésticos: um estudo com base nos dados da Pesquisa Nacional por Amostragem de Domicílios de 2001 e 2005. Rio de Janeiro: IBGE.

Weisner, T. S., \& Gallimore, R. (1977). My brother's keeper: Child and sibling caretaking. Current Anthropology, 18(2), 169-190.

Recebido em: 17/12/2012

Versão final em: 6/9/2013

Aprovado em: 18/10/2013 


\section{Anexo}

Questionário de Tarefas Domésticas e de Cuidado de Irmãos*

INSTRUÇÕES: Por favor, responda as seguintes questões sobre as tarefas que você realiza em casa. Responda a cada pergunta circulando a sua resposta. Escolha UMA resposta para cada pergunta.

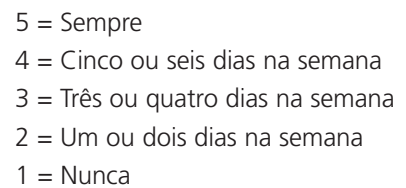

\begin{tabular}{|c|c|c|c|c|c|c|}
\hline 1 & Com que frequência você lava roupas? & 1 & 2 & 3 & 4 & 5 \\
\hline 2 & Com que frequência você toma conta dos seus irmãos menores? & 1 & 2 & 3 & 4 & 5 \\
\hline 3 & Com que frequência você limpa o chão da sua casa? & 1 & 2 & 3 & 4 & 5 \\
\hline 4 & Com que frequência você dá banho nos seus irmãos menores? & 1 & 2 & 3 & 4 & 5 \\
\hline 5 & Com que frequência você limpa o banheiro da sua casa? & 1 & 2 & 3 & 4 & 5 \\
\hline 6 & Com que frequência você cozinha para os seus familiares? & 1 & 2 & 3 & 4 & 5 \\
\hline 7 & Com que frequência você tira o pó dos móveis da sua casa? & 1 & 2 & 3 & 4 & 5 \\
\hline 8 & Com que frequência você leva ou busca seus irmãos menores na escola ou em outra atividade extraclasse? & 1 & 2 & 3 & 4 & 5 \\
\hline \multirow[t]{2}{*}{9} & Com que frequência você serve comida aos seus irmãos menores? & 1 & 2 & 3 & 4 & 5 \\
\hline & Você realiza tarefas domésticas diárias? & \multicolumn{2}{|c|}{$\mathrm{N}$} & \multicolumn{2}{|c|}{$\mathrm{S}$} & \\
\hline 10 & Que idade você tinha quando começou a fazer tarefas domésticas diárias ou semanais? & & & & & \\
\hline 11 & Que idade você tinha quando começou a cuidar dos seus irmãos menores? & & & & & \\
\hline 12 & Que idade você tinha quando começou a cuidar de si mesmo (se virar sozinho)? & & & & & \\
\hline 13 & Se você não cuida de seus irmãos, quem normalmente toma conta deles? & & & & & \\
\hline
\end{tabular}

Nota: ${ }^{*}$ O primeiro fator, que mede cuidado entre irmãos, é formado pelos itens 2, 4, 8 e 9 . O segundo fator, que mede tarefas domésticas, é formado pelos itens $1,3,5,6$ e 7 
